INFORMATION

\title{
Les investissements dans le secteur médical face à la crise financière
}

\author{
T. Fautré \\ Président, division Financial Services de Siemens, France
}

D ans une conjoncture économique toujours incertaine, les récentes pressions sur les marchés financiers ont fait ressurgir le spectre de restrictions budgétaires. Cependant, les institutions politiques et économiques qui ont su tirer les leçons de la crise financière de 2008 insistent depuis sur l'importance de maintenir les niveaux d'investissements dans le secteur de la santé. Comme le souligne un rapport de l'OMS ${ }^{1}$ daté de 2010 , les pays doivent plus que jamais travailler sur l'optimisation des ressources disponibles au risque de voir les disparités dans l'accès aux soins s'accroître. Même en période de crise et face à des arbitrages financiers de plus en plus fréquents, des possibilités de développement et d'amélioration des services de santé existent. Selon ce rapport, 20 à 40\% des dépenses de santé auraient pu être allouées à la création d'une couverture universelle ${ }^{2}$. L'année dernière, le Parlement européen a lui aussi approuvé un rapport ${ }^{3}$ appelant les dirigeants des États membres à soutenir leurs investissements et à faire preuve de plus de discernement dans l'utilisation des ressources budgétaires.

Cet apparent paradoxe entre pressions budgétaires et impératifs de développeseraient "gaspillées » alors qu'elles

ment n'est pas étranger aux laboratoires d'analyses de biologie médicale (LABM) en France. En effet, malgré plusieurs revalorisations à la baisse des tarifs des actes de biologie médicale et dans un contexte global de réduction des marges, l'échéance de l'application de la loi HPST rendant obligatoire l'accréditation de tous les LABM à la norme ISO 15189 d'ici 2016 ne pourra être reportée. Pour relever ce défi de l'accréditation, les directeurs de laboratoires devront se tourner vers des techniques de financement alternatives comme le financement locatif pour les dépenses d'investissements pour ainsi préserver leurs fonds propres pour les investissements humains.

Pourtant, alors que la plupart des gouvernements cherchent à réduire leurs dépenses de santé, la récente étude de la division Financial Services de Siemens (SFS) ${ }^{4}$ n'a fait que confirmer le constat des possibilités de rationalisation des investissements des établissements de santé de plusieurs grandes puissances économiques mondiales telles que la France, l'Allemagne, le Royaume-Uni, la Chine et la Russie : l'étude a en effet révélé qu'environ 21 milliards d'euros, dont 2,3 milliards en France, étaient immobilisés ou « gelés » dans l'achat au comptant d'équipements

${ }^{1}$ Organisation Mondiale de la Santé, Rapport sur la santé dans le monde : Le financement des systèmes de santé, le chemin vers une couverture universelle, 2010.

2 Organisation Mondiale de la Santé, Rapport sur la santé dans le monde : Le financement des systèmes de santé, le chemin vers une couverture universelle, 2010.

${ }^{3}$ Edite Estrela, Rapport sur la réduction des inégalités en matière de santé, Commission européenne de l'environnement, de la santé publique et de la sécurité alimentaire, 8 février 2011.

4 Siemens Financial Services, Melting the Iceberg, mai 2012.

${ }^{5}$ Organisation Mondiale de la Santé, Rapport sur la santé dans le monde : Le financement des systèmes de santé, le chemin vers une couverture universelle, 2010. médicaux à obsolescence rapide. Ces investissements auraient pu faire l'objet d'un financement locatif qui répond aux contraintes techniques et budgétaires des établissements de santé tout en permettant un renouvellement fréquent des équipements concernés.

Ces fonds propres immobilisés sont donc indisponibles pour d'autres dépenses et investissements stratégiques. L'Organisation Mondiale de la Santé insiste notamment sur l'importance d'investir dans les dernières technologies afin de répondre aux attentes des patients et des pouvoirs publics qui souhaitent renforcer la qualité et justifier le coût des prestations délivrées par les établissements de santé. En effet, dans le cas de nombreuses pathologies, I'utilisation des dernières technologies permet une prise en charge précoce des patients grâce à un diagnostic plus précis qui engendre une réduction des durées d'hospitalisation et des traitements moins onéreux ${ }^{5}$.

L'acquisition et le renouvellement fréquent des équipements médicaux fait partie des mesures phares visant à l'atteinte d'un modèle économique viable pour les établissements de santé, alliant efficacité technique et rentabilité. À l'échelle mondiale, pour que le secteur de la santé puisse tirer parti des ressources financières disponibles, des organismes tels que l'OMS encouragent les établissements de santé à optimiser les contrôles qualité afin d'éliminer les dépenses inutiles et préconisent des mesures de financement alternatives. À l'avenir, il est indéniable que les gouvernements devront s'inscrire dans cet état d'esprit et se montrer de plus en plus inventifs dans leurs recherches de financement. • 